\title{
Evaluation of the role of the Treponema pallidum immobilisation test in Britain
}

\author{
M S SPROTT, J B SELKON, AND R H TURNER \\ From the Regional Public Health Laboratory, Newcastle General Hospital, Newcastle upon Tyne
}

SUMMARY After specimens of sera from 100000 patients had been screened by a reagin and Treponema pallidum haemagglutination assay (TPHA) $2 \cdot 7 \%$ required further examination. The fluorescent treponemal antibody-absorption (FTA-ABS) test confirmed either a negative or positive TPHA result in $95 \%$ of the samples, so that no further investigations were required. This accounted for $99 \%$ of the specimens submitted to the laboratory. Repeat tests on a further sample resolved many of the remaining discrepancies. Only $0.05 \%$ of patients, in whom repeat tests confirmed a positive TPHA but a negative FTA-ABS result, benefited from a TPI test.

\section{Introduction}

Infection with Treponema pallidum causes a wide variety of antibodies to be produced. Tests for these fall into two main groups: the first detects the nonspecific antitreponemal antibodies, and includes the tests for antilipoidal and treponemal group-reactive antibodies; the second includes the specific $T$ pallidum tests. The first work on such a test was described by Nelson and Mayer in 1949, ${ }^{1}$ who developed the Treponema pallidum immobilisation (TPI) test. This made available a test which was highly specific for treponemes of the syphilis-yawsbejel group. Its most useful application was considered to be for patients with suspected latent syphilis by differentiating true syphilitic reactions in tests using lipoidal antigen from biological falsepositive reactions. The TPI test is technically difficult and was later superseded by the fluorescent treponemal antibody (FTA) test and the more specific FTAABS test. In $1965^{2}$ the Treponema pallidum haemagglutination assay (TPHA) provided the first specific serological test which could be read with the naked eye.

Which of the specific tests should be used in the diagnosis and monitoring of syphilis is frequently debated. Rein et $a^{3}$ concluded that the TPI test failed to add important diagnostic information to that obtained by the FTA-ABS test and seriously questioned the need for the former's continued use.

Address for reprints: Dr J B Selkon, Public Health Laboratory, Institute of Pathology, General Hospital, Westgate Road, Newcastle upon Tyne NE4 6BE

Accepted for publication 23 November 1981
The aim of this study was to determine the value of the TPI test in the serological examination for syphilis in Britain.

\section{Materials and methods}

During the period February 1977 to April 1980 specimens of sera from 100000 patients were submitted to the Public Health Laboratory, Newcastle upon Tyne, for screening for syphilis by the Venereal Disease Research Laboratory (VDRL) and the TPHA tests. Of these sera, $2728(2 \cdot 7 \%)$ gave positive results in one or other of these tests and were further examined by the FTA-ABS and TPI tests.

In cases in which no clinical information was obtained from the request form submitted with the specimen the physician in charge of the patient was requested to supply relevant details and the ultimate diagnosis.

The Don Whitley TPHA test (Don Whitley Scientific Ltd, Shipley, West Yorkshire) was performed with microtitre equipment according to the manufacturer's instructions using the recommended final serum dilution of $1 / 80$. The TPI, VDRL, and FTA-ABS tests were performed as described ${ }^{4}$; the only notable difference was the use of Wellcome sorbent in the FTA-ABS test at a working titre of $1 / 5$.

\section{Results}

The $2728(2 \cdot 7 \%)$ serum samples giving positive results in either the VDRL or TPHA test or both were examined by the FTA-ABS and TPI tests. The FTA-ABS test confirmed the results of the TPHA 
test in 2611 specimens but discrepancies were found in 117 .

TPHA-NEGATIVE/FTA-ABS-POSITIVE RESULTS

Sera from 68 patients gave TPHA-negative and FTAABS-positive results and were examined by the TPI test; of these, seven gave positive results and the patients were regarded as having had treponematosis. Of the 61 patients whose sera were TPI-negative, 42 (69\%) had clinical evidence of past or recent treponemal disease and $13(21 \cdot 3 \%)$ had no convincing evidence. For six $(\mathbf{9} \cdot \mathbf{8 \%})$ patients clinical information was inadequate to allow a definitive diagnosis to be made. Thus the TPI test contributed very little to elucidating the discrepancy between sera with negative TPHA and positive FTA-ABS results.

TPHA-POSITIVE/FTA-ABS-NEGATIVE RESULIS

Sera from 49 patients gave TPHA-positive and FTAABS-negative results. On examination by the TPI test six (12\%) gave positive results (table); four patients had evidence of past or recent treponemal disease but two had none. Of the 43 patients whose sera were TPI-negative, $13(26 \%)$ had evidence of past or present treponemal disease but most $(30$, $62 \%$ ) had no clinical or previous serological evidence to suggest the diagnosis of a treponematosis. Nine of these had a second specimen retested and in five all the serological tests including the TPHA gave negative results. Thus, the TPI test again contributed little to elucidating the discrepancy between sera with

TABLE Discrepancies between the TPHA and FTA test results

\begin{tabular}{lccc}
\hline & \multicolumn{2}{l}{ No of sera } \\
\cline { 2 - 4 } Tests & $\begin{array}{l}\text { Total } \\
\text { reactive }\end{array}$ & \multicolumn{2}{c}{ TPl results } \\
\hline TPHA - & 68 & + & - \\
FTA + & 69 & 7 & 61 \\
TPHA + & 49 & 6 & 43 \\
Total & $117^{*}$ & 13 & $1(14$ \\
\hline
\end{tabular}

+ Positive; - negative

*4. $3 \%$ of 2728 sera

TPHA $=T$ pallidum haemagglutination assay; FTA = fluorescent treponemal antibody (test).
M S Sprott, J B Selkon, and $R$ H Turner

positive TPHA and negative FTA-ABS results, except $t_{\Phi}^{\frac{\Phi}{D}}$ that the negative TPI results usually confirmed the diagnosis that the patient did not have a treponematosis.

\section{Discussion}

In this study the discrepancies in which the TPHA $\frac{\overline{\bar{S}}}{\sqrt{\frac{\sigma}{2}}}$ test result was negative and that of the FTA-ABS test $\stackrel{\odot}{\mathscr{Q}}$ positive nearly all occurred in patients with earlyo treponemal infection. Since the TPI test is likely to be negative at this stage of the disease its performance adds no additional information and tests $\overrightarrow{\vec{\omega}}$ repeated on a further specimen would be of greater diagnostic value.

Discrepancies in which the TPHA test result was positive and that of the FTA-ABS test negative dide occur in a small number of patients with a past $\vec{A}$ history of a treponematosis. Most patients in this group, however, had no such history or any other 3 evidence of a treponemal infection. In these patientsa negative TPI test result was useful supportive evidence of this diagnosis. Some of these patients $\widehat{D}$ gave completely negative serological test results when $\overrightarrow{0}$ repeat specimens were examined, so that only those patients (estimated at $0.05 \%$ of the total population screened) who yielded persistently positive TPHAO but negative FTA-ABS test results benefited from additional investigation by the TPI test.

We wish to thank the staff of the serology department for their co-operation in this study.

\section{References}

1. Nelson RA, Mayer MM. Immobilization of Treponema pallidum in vitro by antibody produced in syphilitic infection. J Exp Med 1949; 89:369-93.

2. Rathlev T. Haemagglutination test utilising antigens from $O$ pathogenic and apathogenic Treponema pallidum. WHO/VDTIRES/77;65.

3. Rein MF, Banks GW, Logan LC, et al. Failure of the Treponema pallidum immobilization test to provide additional diagnostic information about contemporary problem sera. Sex $\mathrm{N}$ Transm Dis 1980;7:101-5.

4. Wilkinson AE. Serological tests for syphilis. In: Public Health $\mathrm{N}$ Laboratory Service Monograph Series No 1. London: HMSO, 1972:1-24. 